\title{
Appendix Neuroendocrine Tumor
}

National Cancer Institute

\section{Source}

National Cancer Institute. Appendix Neuroendocrine Tumor. NCI Thesaurus. Code C96422.

A well differentiated, low or intermediate grade tumor with neuroendocrine differentiation that arises from the appendix. 